\title{
El aumento del uso de los medios de comunicación masivos por crisis del Coronavirus
}

\section{The increase in the use of mass media due to the Coronavirus crisis}

\section{Como citar el artículo}

Espinoza, L. (2020). El aumento del uso de los medios de comunicación masivos por crisis del Coronavirus. Revista Naturaleza, Sociedad y Ambiente, 7 (1), 93-101. DOI: https://doi.org/10.37533/cunsurori.v7i1.54

Linda Evelin Espinoza Recinos

\section{Centro Universitario de Oriente, Universidad de San Carlos de Guatemala}

Recibido: 29 de abril de 2020 / Aceptado: 09 de septiembre de 2020

Disponible en internet el 10 de noviembre de 2020

*Autor para correspondencia, correo electrónico: misslinda974@gmail.com

\begin{abstract}
Resumen
El presente año ha sorprendido a la humanidad con la aparición de una enfermedad altamente contagiosa llama Covid-19. Ante la posibilidad de frenar el avance de dicha enfermedad por el mundo con la práctica de medidas higiénicas y sanitarias, se suspendieron toda clase de actividades colectivas. Como resultado del confinamiento y del distanciamiento social, la mayoría de las personas ha recurrido al Internet como una forma de mantener el contacto con el mundo exterior sin poner en riesgo la salud. Esto hace más propensas a las personas de todas las edades a peligros tales como: campañas de desinformación, ciberataques, mal uso de datos personales y acciones de manipulación, ya que en el Internet las regulaciones brillan por su ausencia debido a que las gigantes tecnológicas esquivan la responsabilidad sobre los contenidos que circulan a través de sus plataformas. Esta situación lleva a repensar el papel que todos los involucrados tenemos en la actividad humana en Internet y lograr la colaboración entre las grandes corporaciones que comparten contenidos en Internet, acuerdos de cooperación entre países para regular dichos contenidos, pero también responsabilidad individual de impregnar de carácter bioético la red y definir buenas prácticas basadas en la cibernética de segundo orden.
\end{abstract}

Palabras clave: contacto, regulaciones, innovación, responsabilidad, colaboración

\section{Abstract}

This year has surprised humanity with the appearance of a highly contagious disease called Covid-19. Faced with the possibility of halting the advance of this disease around the world through the practice of hygiene and health measures, all kind of collective activities were suspended. As a result of social confinement and alienation, most people have turned to the Internet as a way to maintain contact with the outside world without putting their health at risk. This makes people of all ages more vulnerable to dangers such as: disinformation campaigns, cyber-attacks, misuse of personal data and manipulative actions, since regulations are conspicuously absent on the Internet as technological giants avoid responsibility for the content circulating through their platforms. This situation leads to a rethinking of the role that all of us, who are involved in human activity on the Internet have to play and to achieve collaboration between the large corporations that share content on the Internet, cooperation agreements between countries to regulate such content, but also individual responsibility for impregnating the network with a bioethical nature and defining good practices based on second-order cybernetics.

Keywords: contact, regulations, innovations, responsibility, collaboration 


\section{Introducción}

Los medios de comunicación social han ocupado un puesto relevante en la vida de las personas y lo siguen ocupando en la actualidad. Hay muchos medios de comunicación social. El termino medios de comunicación tradicionales se refieren a cualquier medio de comunicación masivo que apareció antes de la invención del Internet y las redes sociales. Unos de los más antiguos tipos de medios de comunicación social son los impresos. Esto incluye los periódicos, y las vallas publicitarias. Con el desarrollo de la tecnología, se agregaron la radio y la televisión a los medios de comunicación tradicionales.

Hoy, millones de personas usan las redes sociales para esparcir sus ideas y mensajes. Las redes sociales se refieren a cualquier sitio "web" o tecnología que permita a los usuarios crear y compartir información con una audiencia específica. También se les puede llamar medios de comunicación en masa, que es un tipo de medio de comunicación que alcanza un número muy grande de personas y que incluye los medios de comunicación tradicionales y las redes sociales. Hoy podemos decir que la tecnología ha crecido y que las maneras en las cuales recibimos los mensajes ha cambiado. Aunque leemos textos también miramos fotos, datos y cuadros. Miramos videos y escuchamos la radio, podcasts y otras grabaciones de audio. Para que podamos procesar correctamente toda esa información se necesita una adecuada alfabetización.

\section{La alfabetización en los medios de comunicación social}

La alfabetización según el contenido: "Can you separate fact from fiction?" del MOOC "English for Media Literacy" (2019) es la habilidad de leer, entender, analizar y crear usando el lenguaje escrito. Parte de la alfabetización es analizar texto. Analizar significa pensar sobre algo cuidadosamente para entenderlo. Alguien que es culto en los medios de comunicación abre el periódico o ve una publicación (un "post") en las redes sociales sabe hacer preguntas importantes sobre el mensaje y las personas que hicieron ese mensaje.

Las personas son inundadas con mensajes, no importa si llegan en línea, por televisión o por periódico.

La alfabetización sobre medios de comunicación se refiere a entender cómo y porqué los mensajes son propagados. Todo inicia con las preguntas correctas: ¿quién creó este mensaje?, ¿qué palabras o imágenes usaron y por qué? Y ¿cómo se supone que este mensaje me hace sentir?

a) ¿Quién creó el mensaje? Todos los mensajes son hechos por personas. Estas personas hacen elecciones sobre lo que incluirá o no un mensaje. Las imágenes y videos que vemos y las palabras que leemos nos cuentan una historia. Por lo cual es importante pensar en qué opciones fueron hechas y como afectan o cambian la historia que se cuenta.

b) ¿Qué técnicas usaron para atraer mi atención? Cuando los medios crean un mensaje usan ciertas técnicas para capturar la atención de su audiencia. Unas veces usan el humor - la comedia, también usan ciertos símbolos y palabras. La meta es que el usuario siga leyendo y viendo el mensaje en el futuro. 
c) ¿Qué puntos de vista y valores son incluidos u omitidos del mensaje? Por ejemplo, piensa en una persona que quiere participar en las elecciones para la presidencia de su país. Un mensaje puede decir que esa persona podría ser un gran presidente. Mientras otro podría decir que sería un terrible presidente. ¿Cómo puede la misma persona ser al mismo tiempo un excelente y un terrible presidente? No hay que olvidar que las organizaciones que crearon esos mensajes tienen creencias políticas y valores diferentes. Sin olvidar que las ideas o creencias que se omiten nos hacen perder de vista parte de la historia que los medios nos quieren hacer creer que no son importantes.

La alfabetización sobre medios de comunicación te enseña a pensar cuidadosamente sobre la información. Aprendes a hacer las preguntas correctas, mirar de forma diferente los puntos de vista y las conexiones entre nuevas y viejas ideas y estas preguntas no son solo importantes cuando miras televisión o revisas tu teléfono inteligente. El pensamiento crítico te ayuda muy bien en otras partes de la vida como el aula y la oficina. Recuerda que escuchas y ves más mensajes en los medios en un día que otras personas lo hicieron en el pasado. Debes poner mucha atención a lo que lees, miras y escuchas, ser consciente de su propósito así tomarás mejores decisiones y distinguirás entre los datos reales y la ficción.

La alfabetización en medios de comunicación te ayuda en dos destrezas necesarias para ser un ciudadano de una democracia: pensamiento crítico y autoexpresión sin caer en la trampa de las noticias falsas que son tan comunes en la actualidad.

\section{Tiempo dedicado al Internet}

En 2014 el promedio que las personas en América gastan en el Internet es de 7.4 horas. La gente de Filipinas, un país que se ubica en el Océano Pacifico ganó el primer lugar. Éstas personas gastaron 9.6 horas por día en Internet desde sus computadoras de escritorios, laptops y dispositivos móviles. De hecho, la gente ha estado gastando una enorme cantidad de tiempo en el Internet a través del mundo. Como resultado del confinamiento y del distanciamiento social debido al Covid-19, todavía más personas han recurrido al Internet como una forma de mantener el contacto con el mundo exterior sin poner en riesgo la salud.

El Internet ofrece acceso a mucha información las 24 horas del día. Las redes sociales son los contenidos más populares, pero también la gente gusta mucho de ponerse al día con las noticias del momento.

Las redes sociales son estructuras sociales compuestas de grupos de personas, las cuales están conectadas por uno o varios tipos de relaciones y mediadas por plataformas tecnológicas que constituyen el canal de intercambios que posibilitan las interacciones definidas, según Cobo y Romaní (2007) las redes sociales describen "aquellas herramientas diseñadas para la creación de espacios que promueven o faciliten la conformación de comunidades e instancias de intercambio social".

Actualmente, el uso de redes sociales es una actividad normal que cualquier persona puede realizar desde su dispositivo móvil, tableta o computadora. Las redes sociales más populares son: "Facebook, Twitter, Ins- 
tagram, Tmblr, Reddit, Linkedln and YouTube". El hecho es que, los seres humanos nos hemos vuelto adictos a este tipo de interacciones, debido exactamente a que dichas redes sociales están diseñadas para volverse adictivas.

Según Adam Alter (2018) para las grandes empresas de la tecnología masiva tales como: Google, Facebook, Netflix, entre otras existe una correlación directa entre atención y crecimiento, por lo cual éstas están diseñadas precisamente para volverse altamente adictivas. Aunque las tendencias adictivas aparecieron mucho antes que el Internet, los teléfonos inteligentes y las redes sociales han ampliado grandemente dichas tendencias.

- Recompensas variables: las redes sociales son como máquinas tragamonedas. Cada notificación, cada foto nueva, cada me gusta, es único y cambian a cada momento. La forma en que están diseñadas es muy difícil de resistir.

- Distracción: las redes sociales no son predecibles. A los seres humanos nos aburre la predictibilidad de nuestras vidas, es por eso que las redes sociales nos distraen y crean un circulo de retroalimentación que se vuelve más llamativo cuanto más lo usamos.

- Detener las señales: Las redes sociales usan vertiginosamente el video con desplazamiento infinito, es decir, el contenido nunca se detiene. Esto hace que cinco minutos se conviertan en treinta, sin que el usuario se dé cuenta.

- Métrica de vanidad: en las redes sociales puedes conseguir pequeñas victorias tales como: tienes más "likes" que tu mejor amiga, sobrepasaste a todos tus amigos por el "Snapstreak" más largo, tu foto o publica- ción tiene más comentarios que los de otras personas conocidas. Cada una de esas pequeñas victorias no significan nada, pero nos dan un golpe de dopamina cada vez, y su frecuencia cada vez mayor nos lleva a pasar más tiempo y esforzarnos aún más para alcanzar nuestros objetivos triviales de forma regular.

\section{Las redes sociales, una receta para la adicción}

Las redes sociales son una receta para la adicción, una muy potente por cierto y la parte más preocupante es que estos factores hacen que las personas eviten las interacciones cara a cara, pasen menos tiempo con familiares y amigos, e incluso arriesguen sus vidas (son conocidos los casos de jóvenes que arriesgan sus vidas por tomar "selfies" en lugares sumamente peligrosos para lograr la atención de miles de seguidores). Y aún hay más, estas adicciones nos hacen perder tantos momentos que nunca tendremos la oportunidad de revivir. Nos perdemos los mejores momentos de la vida real por pasar demasiado tiempo en los contenidos del Internet.

Pensando en esta adicción a los dispositivos electrónicos, se podría decir que la falta de conciencia sobre el tiempo que invertimos y la invasión en nuestras vidas que estos artefactos provocan, es la causa que más nos debería alarmar sobre el uso y abuso de ellos. Para poder ilustrar esa falta de conciencia sobre el tiempo que nos roba, pensemos por ejemplo que en promedio una persona normal revisa su teléfono móvil cada seis minutos (150 veces al día), la sola presencia del teléfono inteligente, aunque esté apagado, reduce nuestra capacidad cognitiva y, además, en menos de dos décadas, nuestro tiempo 
cara a cara con familiares y amigos ha disminuido en casi un treinta por ciento.

\section{Facebook, una de las redes sociales más populares}

Facebook es una red social creada por Marck Zuckerberg, quien de acuerdo con los datos de Bloomberg (compañía estadounidense de asesoría financiera), es la quinta persona más rica del mundo. Esta red social hace mucho dinero con nuestros datos mientras nos permite compartir, comentar y conectarnos con quien queramos. También tiene la capacidad de rastrear nuestra ubicación, nuestros desplazamientos y costumbres para almacenar esos datos y poder recrear un retrato robot lo más parecido a cada usuario. Recordemos que cada uno de nosotros somos dueños de nuestra privacidad y es precisamente eso lo que sacrificamos cuando usamos la red de tecnología móvil.

Con la reciente cuarentena por el Covid-19, Facebook tuvo un incremento de 105 millones de usuarios solo en el primer trimestre del 2020. Si tomamos en cuenta que la población mundial es de aproximadamente 7,800 millones de personas, que la mitad de esas personas no tienen acceso a Internet y que Facebook está prohibido en China y otras naciones, podemos darnos cuenta que su alcance activo de esta red social es absoluto. Aunque Facebook es una de las redes sociales más usadas, no podemos ignorar que existen otras muy importantes que han ganado popularidad entre los usuarios. Twitter, Tumblr and YouTube también tienen altos números de usuarios registrados obteniendo noticias de sus sitios web. Doug Bernard opina que el número de personas usando más de un sitio para obtener noticias es de solo el $26 \%$ y solo el $10 \%$ dice usar tres o más.
Los investigadores, de hecho, han encontrado que las noticias en de las cinco redes sociales más populares parecen ir dirigidas a diferentes tipos de personas. La gente que busca noticias en Twitter es la más joven. Los usuarios de las noticias en Facebook son en su mayoría del sexo femenino. Las personas con grados académicos buscan noticias en Linkedln.

\section{Las redes sociales y los rituales}

Pero el aumento del uso de las redes sociales no es la única consecuencia de la crisis del coronavirus, también dicha crisis está acabando con algo tan importante como lo son los rituales. Se está prohibiendo, por razones sanitarias, hasta el darse la mano. La pandemia está dando lugar a una sociedad de la cuarentena en la que se está perdiendo toda experiencia comunitaria. Aunque seguimos conectados digitalmente, seguimos comunicándonos, pero sin ningún acercamiento social que nos haga realmente felices. Incluso, antes del Covid-19, lo que conocemos y entendemos como comunidad ya se estaba extinguiendo con la desaparición de los rituales.

El filósofo alemán vivo más leído del mundo, Byung-Chul Han, señala que los rituales anclan la comunidad en el cuerpo ya que en ellos el cuerpo es un escenario en el que se inscriben los secretos, las divinidades y los sueños. La cortesía es un ejemplo de esos rituales. Cada día celebramos menos fiestas comunitarias, cada uno se celebra sólo a sí mismo. Debemos inventar nuevas formas de acción y juego colectivo que creen comunidad. La comunidad es fuente de felicidad. También la libertad tiene sus raíces en lo comunal, libertad y amigo tienen una etimología común. La libertad es la manifestación de una relación plena. Es por ello que debe- 
ríamos redefinir la libertad a partir de la comunidad. Por lo cual, según Han (1959), al desaparecer la comunidad ira desapareciendo también parte de nuestra libertad.

\section{La publicidad en los medios de co- municación social}

Pero aún hay más, en las redes sociales también vamos a ser bombardeados por otra herramienta muy popular para vendernos cualquier producto o servicio, "la publicidad". Las empresas de publicidad, según Nancy Bollinger (Directora Creativa), estudian a las personas, saben datos demográficos, la edad, residencia, ingresos, e información psicográfica realmente útil tales como estilo de vida, pasatiempos, intereses para captar clientes potenciales. Esto lo pueden hacer de varias maneras, por ejemplo, si ya hay clientes, ellos son la fuente de información más confiable. Si es un nuevo negocio, consideras cual será tu audiencia destinataria para encontrarlos y motivarlos a comprar.

La publicidad es motivación. No les venden un producto a las personas sino un estilo de vida, no satisfacen una necesidad sino crean necesidades para luego satisfacerlas. Por ejemplo, en 1930, no era común que las mujeres lucieran anillos de diamantes. En esa época De Beers Diamonds tuvo un exceso de diamantes de las minas del Sur de África y fue cuando inició una campaña para hacer creer a los hombres que la mejor manera de demostrar su amor por una mujer era regalando un hermoso anillo de diamantes, cuánto más grande y cara fuera la joya más grande era el amor que representaba y fue así como se convirtió en una tradición.

\section{La cibernética de segundo orden y el abordaje del tiempo y el uso del Internet}

Primero quiero que se tome en consideración que el hombre siempre ha tratado de dar una explicación al orden del mundo. Heráclito de Éfeso, Anaximandro, entre otros, descubrieron el mundo de la experiencia dando cuenta del orden del cosmos. Posteriormente René Descartes, llegó a la conclusión que para entender un fenómeno había que reducir a partes y procesos elementales, mirarlos aisladamente, para luego resolver la dificultad y creó la dualidad entre mente y cuerpo. Gracias al método de Descartes los eventos de análisis se redujeron a dos variables: causa-efecto.

Los términos científicos que se venían dando se referían a los efectos lineales, aunque el pensamiento de sistemas ya existía en Aristóteles, desaparece con Descartes y la realidad comienza a ser desmembrada y reducida a una creciente fragmentación.

A partir de la Segunda Guerra Mundial se comienza a consolidar un pensamiento cada vez más totalizador y menos fragmentado con Ludwig Von Bertalanffy. El emprendió la búsqueda de la unidad en la diversidad, dando origen a una nueva era en la ciencia, la que llamó sistemas. Bertalanffy inició el desarrollo del pensamiento sintético, el cual sostiene que todo sistema pertenece a un sistema más amplio (un sistema mayor) y que todo sistema es un todo organizado. Lo interesante es que la Teoría de sistemas estaba más interesada en integrar las cosas que en aislarlas, además propone unos modelos de sistema general como intento de unificar el conocimiento científico. 
La Cibernética se volvió en piedra angular de la Teoría de Sistemas, y juntos, Cibernética y Teoría de sistemas ofrecen al campo de las ciencias una nueva cosmovisión. En resumen, la Cibernética se basa en el supuesto según el cual las funciones de control, intercambio de información y procesamiento de la información, siguen los mismos principios, independientemente de que se apliquen a máquinas, organismos o estructuras sociales.

La Cibernética de Primer Orden es la ciencia que estudia los sistemas complejos: máquinas, organismos biológicos, grupos humanos, a través de los mecanismos de regulación interna y con especial atención a sus finalidades. En ella se postula que el observador se mantiene fuera del fenómeno observado, dando origen a la suposición de que aquél es capaz de manipular o controlar unilateralmente el sistema que está observando.

La Cibernética del Segundo Orden es el estado de las condiciones en que los sistemas humanos toman conciencia de los mecanismos de regulación interna y de sus finalidades, transformando de ese modo sus mismos mecanismos de regulación y esas finalidades. El observador es consciente, "se observa observar, y con ello transforma radicalmente el carácter de su observación" (Foerster, 1994).

La Cibernética de Segundo Orden es como el manifiesto aplicable a nuestra propia vida, dado que en el diario vivir construimos con el otro una nueva realidad cimentada en la libertad y la responsabilidad. Foerster lo destaca de la siguiente manera: "La cibernética de segundo orden abre un espacio para la reflexión sobre el propio comportamiento y entra directamente en el territorio de la res- ponsabilidad y la ética, fundamentado en la premisa de que no somos descubridores de un mundo exterior a nosotros, sino inventores o constructores de la propia realidad". Asumir esta posición se manifiesta en el quehacer científico, docente, empresarial, pero también en las relaciones de la vida diaria.

Según Jutoram (1994), si uno se considera un observador independiente "puede decir al otro cómo pensar y actuar: Tú debes, tu no debes. Este es el origen de los códigos morales. Si uno se considera un actor participante en el drama de la mutua interacción, del dar y recibir, en la circularidad de las relaciones humanas dada mi interdependencia, sólo puede decirme a mí mismo cómo pensar y actuar: Yo debo, yo no debo este es el origen de la ética".

Desde este punto de vista, soy yo como usuario de Internet quien debe reflexionar sobre la reflexión del uso que le estoy dando a mi tiempo en el Internet, ¿qué redes sociales son las que más utilizo?, ¿Qué tipo de información es la que comparto?, ¿qué tipo de fotografías estoy publicando?, ¿cuánto tiempo de mi vida le dedico a estar conectado/a?, ¿Qué cosas dejo de hacer?, ¿a quién le estoy negando el tiempo que le otorgo al Internet?, ¿qué experiencias dejo de vivir?, ¿es razonable lo que pierdo comparado con lo que gano usando el Internet?, ¿estoy consciente del efecto de la publicidad en mi vida?

Creo que es el momento de hacer una reflexión basada en la cibernética de segundo orden para poder establecer clara y razonablemente hasta donde estoy dispuesto a permitir la pérdida de mi privacidad, la poca convivencia familiar, renunciando a un tiempo que no volverá, el querer impresionar a gente 
que no conozco y que no me quiere, a sumar muchos amigos virtuales pero muy pocos 0 ningún amigo de verdad, a dejar morir los rituales comunitarios, hasta donde soy capaz de distinguir la verdad de la ficción en los textos que leo en las redes, a desarrollar una alfabetización que me ayude a desarrollar mi libertad y mi responsabilidad.

Por una parte, es importante colaborar para que existan regulaciones razonables a lo que se puede y se debe publicar en el Internet, ya que es un espacio que carece de regulaciones. Es sumamente fundamental evitar que el actual formato de innovación y desarrollo "corporativo e industrialista" atropelle y vuelva más precarios los derechos humanos de las poblaciones. Por otra parte, es muy deseable y conveniente la regulación pública para aprovechar mejor las tecnologías en la construcción de sociedades más justas, incluyentes y democráticas. De hecho, hay tres aspectos que degradan los derechos ciudadanos a escala global:

a) La privacidad. Existen varios informes y documentos a nivel internacional que describen un escenario de violación masiva de los derechos a la intimidad/privacidad reconocidos a los pueblos en las Constituciones nacionales y garantizados en instrumentos internacionales. Recordemos el caso de vigilancia masiva y ciberespionaje que algunos Estados-nación han construido a nivel global, que se volvieron de dominio público a través de las filtraciones de Chelsea Mannign y Edward Snowden.

b) Derecho de autor y derecho de copia. Las capacidades de copia se están distribuyendo a nivel internacional de una forma injusta $y$ asimétrica. No está claro aún cómo deben repensarse democráticamente las regulaciones sobre bienes y obras intelectuales, informaciones y datos. El derecho de copia y la disponibilidad de los bienes intelectuales deberían ser construidos y ejercidos como derechos humanos fundamentales.

c) Libertad de expresión y censura. Las libertades de expresión de la ciudadanía no pueden estar secuestradas por las condiciones de uso que plantean algunas corporaciones.

d) Debemos luchar por superar la "neutralidad", un concepto diseñado a la medida de los intereses de las grandes corporaciones de Internet. Debemos lograr el isomorfismo (igualdad ante la ley) para que podamos disfrutar justamente de los avances tecnológicos sin renunciar a nuestra privacidad y sin ser víctimas de los abusos que se pueden llevar a cabo por la falta de regulación en la red de redes.

Es lo que Friedman (2018) llama "el surgimiento de una innovación moral basada en valores sustentables, construidos y consolidados a través de la colaboración". Colaboración entre las grandes corporaciones que comparten contenidos en Internet, acuerdos de cooperación entre países para regular dichos contenidos, pero también responsabilidad individual de impregnar de carácter bioético la red y definir buenas prácticas basadas en la cibernética de segundo orden.

\section{Referencias bibliográficas}

Alter, A., 2018. La adicción a las pantallas avanza silenciosa. El país. 23/04/2018. Tecnología. Disponible en https://elpais.com/tecnologia/2018/04/24/actualidad/1524577831_486816.html 
Can You Separate Fact from Fiction? 2019. For the AETeacher Program, sponsored by the U.S. Department of State and administered by $\mathrm{FHI} 360$. This work is an adaptation of Can You Separate Fact from Fiction by ShareAmerica.gov. Adapted content is licensed under the Creative Commons. Disponible en Http:// Creativecommons.org/licneses/by/4.0/

Cobo Romaní C.; Pardo Kuklinski H. Planeta Web 2.0. Inteligencia colectiva o medios de fast food". Grup de Recerca d'Interaccions Digitals, Universitat de Vic. Flacso México. Barcelona. Disponible en https:// www. planetaweb2.0.net/
Foerster, V.,1994. Ética y cibernética de segundo orden. Santafé de Bogotá, pág. 89.

Friedman, T., 2018. Gracias por llegar tarde. Como la tecnología, la globalización y el cambio climático van a transformar el mundo en los próximos años. Barcelona. Deusto.

Han, B., 1959. La desaparición de los rituales. Barcelona, Herder Editorial.

Jutoram, S., 1994. El proceso de las ideas sistémico-cibernéticas. Sistemas familiares. Año 10, No 1. Buenos Aires. Pág. 20-21.

\section{Sobre autora}

\section{Linda Evelin Espinoza Recinos}

Secretaria Bilingüe, Licenciada en Pedagogía y Administración Educativa y Maestra en Investigación, cuenta con 22 años de experiencia como docente en el nivel medio, ciclo básico y diversificado enseñando Ciencias Sociales y Lenguaje y comunicación L3 (idioma inglés) y 8 años de experiencia laboral en la Carrera de Pedagogía del Centro Universitario JUSAC, de los cuales 6 han sido dedicados a cursos sobre Investigación. Se capacitó como Tutor Virtual en la Universidad Galileo. Actualmente se encuentra cursando el segundo semestre del Doctorado en Investigación en Educación en el Centro Universitario CUNORI.

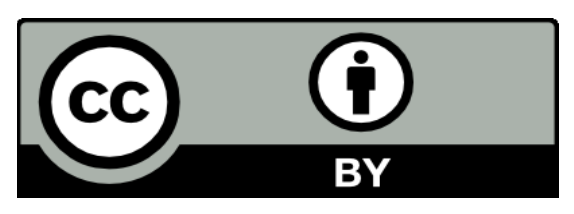

Este texto está protegido por una licencia CreativeCommons 4.0.

Esta licencia permite que otros distribuyan, mezclen, adapten y desarrollen su trabajo, incluso comercialmente, siempre y cuando le den crédito por la creación original. 